\title{
10-kHz spinal cord stimulation treatment for painful diabetic neuropathy: results from post-hoc analysis of the SENZA-PPN study
}

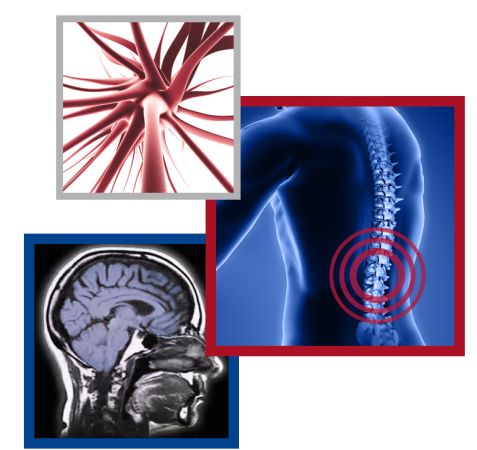

\author{
Vincent Galan 1 , James Scowcroft' ${ }^{2}$, Paul Chang ${ }^{1}$, Sean $\mathrm{Li}^{3}$, Peter Staats ${ }^{3}$, Anand Rotte ${ }^{4}$ (D) \& \\ Jeyakumar Subbaroyan*,4 \\ ${ }^{1}$ Georgia Pain Care, Stockbridge, GA 30281, USA \\ ${ }^{2}$ KC Pain Centers, Lee's Summit, MO 64086, USA \\ ${ }^{3}$ Premier Pain Centers, Shrewsbury, NJ 20852, USA \\ ${ }^{4}$ Nevro Corp., Redwood City, CA 94065, USA \\ *Author for correspondence: Tel.: +1 650433 2726; Fax: +1 650251 9415; jey.subbaroyan@gmail.com
}

\section{Practice points}

- Significant proportion of diabetic patients develop painful diabetic neuropathy (PDN), a serious complication characterized by progressive, chronic debilitating pain resulting from the damage to peripheral nerves.

- Treatment alternatives are clearly needed for the majority of patients with refractory PDN.

- Current analyses evaluated PDN subset data from prospective, single arm, multicenter, early feasibility study of peripheral polyneuropathy of the upper or lower limbs (SENZA-peripheral polyneuropathy study).

- Results showed that pain relief sustained at 12 months (74\%).

- A total of $86 \%$ of subjects were both responders and remitters at 12 months.

- Subjects had no worsening of neurological deficits following 3 and 12 months of stimulation. Instead, improvement in sensory and/or reflexes was reported in $71 \%$ of subjects.

- All study related adverse events were mild-to-moderate and resolved without sequelae.

- Subjects also had improvements in disability (pain disability index), functioning (global assessment of functioning) and sleep (pain and sleep questionnaire) scores.

- $85 \%$ of the subjects reported they felt 'moderately better', 'better' or 'a great deal better' after 12 months of treatment.

Aim: Previous studies of $10 \mathrm{kHz}$ spinal cord stimulation demonstrated its safety and efficacy for treatment of neuropathic pain of the trunk and/or limbs. This study analyzed data from a subset of subjects with painful diabetic neuropathy enrolled in a prospective, multicenter study of peripheral polyneuropathy with various etiologies. Materials \& methods: Of the eight subjects that had permanent devices, seven attended the 12-month follow-up assessment. Results \& conclusion: At 12 months, 6/7 subjects were treatment responders ( $\geq 50 \%$ pain relief) and had pain remission (visual analog scale $\leq 3.0 \mathrm{~cm}$ ). Worsening of neurologic deficits was not reported in any subject. Instead, 5/7 subjects showed improvements in sensory testing and/or reflexes. These results support further investigation of $10 \mathrm{kHz}$ spinal cord stimulation as a safe and effective treatment for intractable painful diabetic neuropathy.

First draft submitted: 13 May 2020; Accepted for publication: 24 June 2020; Published online:

11 August 2020

Keywords: $10 \mathrm{kHz}$ SCS $\bullet$ painful diabetic neuropathy $\bullet$ painful polyneuropathy $\bullet$ VAS

Nearly $10 \%$ of the US population, 30.3 million people, were estimated to have diabetes in 2017 [1]. A systematic literature review aimed to estimate the global impact of diabetes projected the number of people with diabetes would increase to 642 million by 2040 and the total global health expenditure due to diabetes to be US\$673 billion [2]. A significant proportion of diabetic patients develop painful diabetic neuropathy (PDN), a serious complication characterized by progressive, chronic debilitating pain resulting from the damage to peripheral nerves [3]. An observational study of patients with diabetes conducted in northwest England estimated the prevalence of PDN to be $21 \%$ [4]. 
PDN significantly increases the disease burden of patients with diabetes by decreasing quality of life measures and increasing healthcare resource utilization [5]. Common symptoms of PDN include manifestations of central sensitization and/or neurological deficits like burning pain, numbness, paresthesia, weakness [6] and gait abnormalities [7].

The most common first-line treatments for PDN are anticonvulsants, particularly pregabalin and gabapentin [8,9], despite low response rates of less than 50\% [10]. Both medications have relatively high number to treat and high rates of discontinuation due to side effects [11]. Other recommended pharmacotherapies include tricyclic antidepressants and serotonin-norephinephrine reuptake inhibitors both of which have similar low efficacy [9].

Nonpharmacologic alternatives for patients with PDN include neuromodulation utilizing transcutaneous electric nerve stimulation and paresthesia-based low-frequency spinal cord stimulation (LF-SCS). Although transcutaneous electric nerve stimulation is frequently used in neuropathic pain conditions, the published evidence regarding its efficacy is of low quality [12], and it is currently unknown whether this technology is effective for neuropathic pain. Spinal cord stimulation involves implanting an electrode array in the epidural space and connecting the array to a receiver or pulse generator. This is a minor surgical procedure that has been widely performed in the US for more than 50 years. Traditionally LF-SCS has been used to treat a variety of pain states. More recently, novel frequencies, waveforms and approaches have been used with markedly improved success rates. Studies that evaluated LF-SCS to treat patients with PDN showed that 33-59\% did not achieve pain reductions $\geq 50 \%$ at follow-up [13-15], and nonresponse rates remained over $50 \%$ after 24 months of treatment and reached $64 \%$ at 5 years $[16,17]$. Treatment alternatives are clearly needed for the majority of patients who do not achieve adequate long-term pain relief with traditional LF-SCS.

High frequency SCS at $10 \mathrm{kHz}$, is a paresthesia-independent treatment that has been shown to be effective in treating neuropathic pain [18,19] and pain in the lower limbs [20]. In contrast to LF-SCS, paresthesia-mapping is not necessary during $10 \mathrm{kHz}$ SCS trial or implantation, improving procedural efficiency and avoids paresthesiaassociated discomfort. The following pilot study used data from a prospective, multicenter study of patients with peripheral polyneuropathies (PPN) to evaluate the safety and effectiveness of $10 \mathrm{kHz}$ SCS for treating chronic, intractable pain in subjects with PDN.

\section{Materials \& methods}

This pilot study used data from a prospective, single arm, multicenter, early feasibility study of peripheral polyneuropathy of the upper or lower limbs (PPN study) conducted from November 2015 to August 2016 (approved by Western Institutional Review Board, WA, USA) [21]. Subjects were selected for inclusion in this analysis if they had a clinical diagnosis of PDN of the lower $\operatorname{limb}(s)$, their pain was refractory to conservative medical therapy for 3 months or longer, stable medication regimen and their mean visual analog scale (VAS) pain scores for the upper or lower limbs was $\geq 5 \mathrm{~cm}$ on a $10 \mathrm{~cm}$ scale. Subjects were excluded if they were diagnosed with mononeuropathy of the trunk or if they had previous failed SCS trials for chronic intractable pain.

All study subjects received two epidural octopolar leads that were implanted spanning the T8-T11 vertebral bodies and underwent a 1-week trial stimulation. Trials that produced $\geq 40 \%$ pain relief were deemed successful, and those subjects were implanted with a permanent Senza ${ }^{\circledR}$ system (Nevro Corp., CA, USA). All subjects received stimulation at $10 \mathrm{kHz}$ at $30 \mu$ s pulse width, while amplitude of stimulation and other programming options were customized to maximize pain relief for each subject.

Study investigators were trained via video by a neurologist on administration of neurological assessments that were developed with input from the FDA for a previous study. Neurological assessments were conducted at baseline and follow-up visits including motor testing, sensory testing (light touch and pinprick) and reflex testing (Babinski, patellar tendon and Achilles tendon). All adverse events (AEs) and serious adverse events (SAEs) during the study period were recorded by study investigators.

Outcome measures included pain intensity using VAS scores, pain symptoms using the short-form McGill Pain Questionnaire (SF-MPQ-2), disability using the Pain Disability Index (PDI), function using the Global Assessment of Functioning (GAF), sleep using the Pain and Sleep Questionnaire (PSQ-3), subject-reported global impression of change and clinician-reported global impression of change.

Given the high responder rate from previous studies treating lower limb pain [20,22], the original PPN study used a rule of thumb sample size of 25 subjects to assess the treatment of $10 \mathrm{kHz}$ SCS in this patient population. Given the small number of subjects that presented with PDN in the original study, results are summarized in measures of central tendency (mean, standard error) without any statistical tests. 


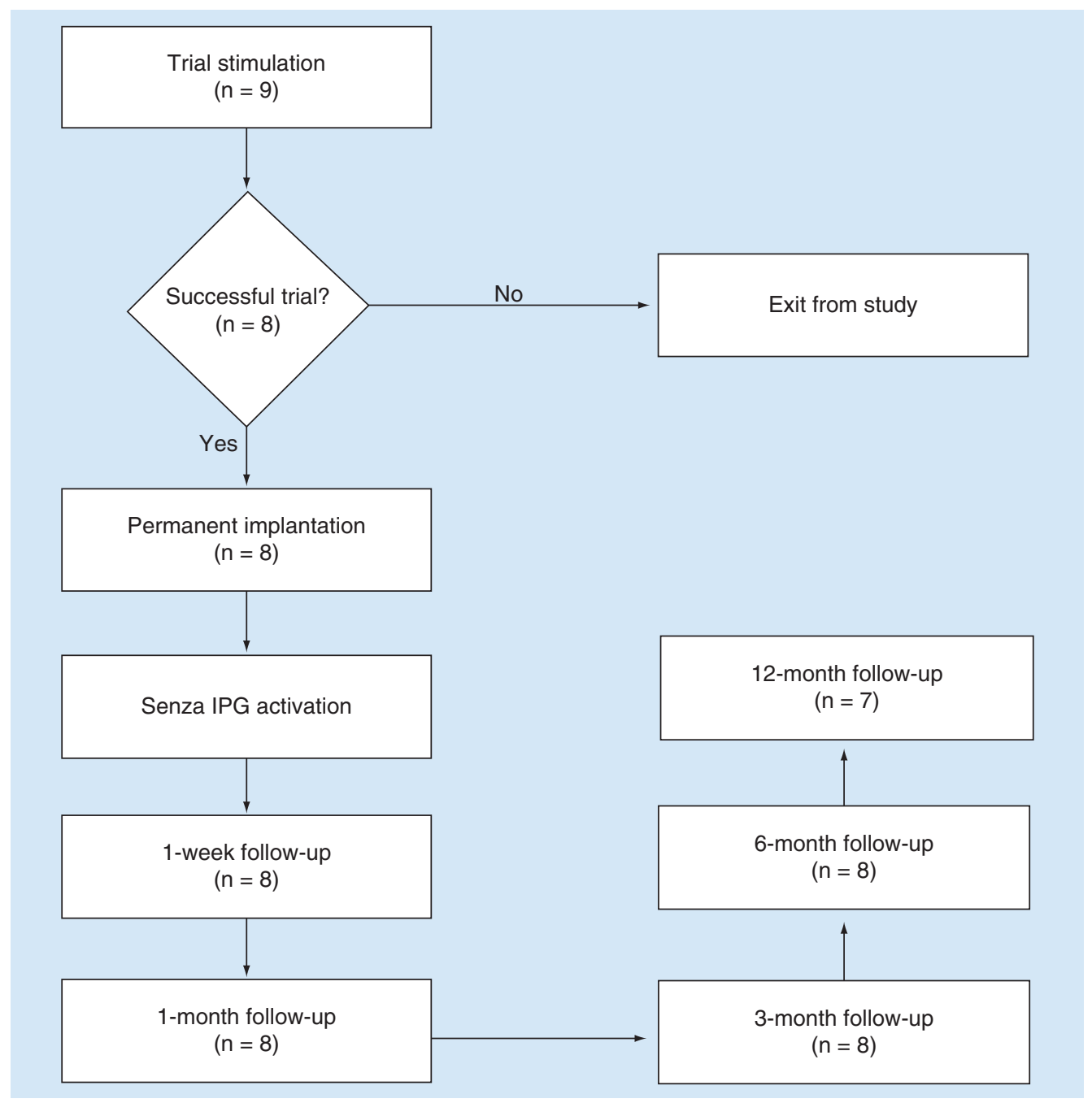

Figure 1. A total of nine subjects met the study criteria and underwent a trial stimulation, eight were implanted with permanent devices and seven received stimulation through the final follow-up visit at 12 months. IPG: Implantable pulse generator.

\section{Results}

A total of nine subjects enrolled in the PPN study had PDN of the lower limbs and were eligible for this sub analysis. Of these, eight $(88.9 \%)$ had successful SCS trial stimulation, with at least $40 \%$ reduction in their pain (mean \pm standard deviation: $86.3 \pm 7.4 \%$, range: 70.4-94.8\%), and received a permanent implantable pulse generator. All implanted subjects attended follow-up visits after 1, 3 and 6 months of stimulation, and seven of eight attended the final follow-up after 12 months of stimulation as shown in Figure 1. Demographics and clinical characteristics of the implanted patients are listed in Table 1.

\section{Pain outcomes}

Mean VAS scores decreased from $8.0 \pm 1.2 \mathrm{~cm}$ at baseline to $2.0 \pm 1.2 \mathrm{~cm}$ after 6 months and $2.1 \pm 2.3 \mathrm{~cm}$ after 12 months of $10 \mathrm{kHz}$ SCS, as shown in Figure 2A. At the 6-month follow-up, seven of eight (87.5\%) subjects were both responders, defined by pain relief of $50 \%$ or more, and remitters with VAS scores of 3.0 or less (Figure $2 \mathrm{~B}$ ). Individual subject responses at 3 months are shown in Figure 2C.

Scores from the SF-MPQ-2 decreased from baseline by 3.3 points after 6 months of stimulation and 2.9 points after 12 months. Similar decreases were observed in all four individual domains: continuous pain, intermittent pain, neuropathic pain and affective descriptors of pain (Figure 3). 
Table 1. Demographic and baseline clinical characteristics of subjects who had trial stimulations.

\begin{tabular}{|lc|}
\hline Characteristics & Subjects $(\mathbf{n}=9)$ \\
\hline - Fender, $\mathbf{n}(\%)$ & \\
\hline - Males & $4(44 \%)$ \\
\hline Age (years) at enrollment & $5(56 \%)$ \\
\hline - Mean \pm SD & \\
\hline - Range & $64.8 \pm 8.8$ \\
\hline Years since diagnosis & $47.0-77.0$ \\
\hline - Mean \pm SD & \\
\hline - Range & $7.2 \pm 5.1$ \\
\hline Ethnicity & $1.0-16.0$ \\
\hline - Non-Hispanic/Latino & \\
\hline Race, $\mathbf{n}$ (\%) & $9(100.0 \%)$ \\
\hline - Black/African-American & \\
\hline - White & $1(12 \%)$ \\
\hline Baseline VAS, mean \pm SD & $8(89 \%)$ \\
\hline Baseline PDI, mean \pm SD & $7.9 \pm 1.1$ \\
\hline PDI: Pain disability index; SD: Standard deviation; VAS: Visual analog scale. & $40.2 \pm 13.1$ \\
\hline
\end{tabular}

\begin{tabular}{|c|c|c|c|}
\hline $\mathbf{n}$ & Description & Relationship & Severity \\
\hline 1 & Pain in extremity (left leg) & Procedure related & Moderate \\
\hline 2 & Hematuria & Not related to study & Mild \\
\hline 3 & Erectile dysfunction & Not related to study & Mild \\
\hline 4 & Implant site seroma & Procedure related & Mild \\
\hline 5 & Hepatic failure & Not related to study & Severe \\
\hline 6 & Clostridium difficile infection & Not related to study & Mild \\
\hline 7 & Abdominal pain & Not related to study & Mild \\
\hline 8 & Intra-abdominal hemorrhage & Not related to study & Mild \\
\hline
\end{tabular}

\section{Safety}

Neurological assessments

Baseline neurological assessments showed improvement or maintenance after the trial stimulation and following 3 and 12 months of stimulation, as shown in Figure 4. None of the subjects showed evidence of worsening neurological functioning in any domain, and all of the observed improvements were in the sensory and reflex assessments, rather than motor assessments, at the 3-month and 12-month follow-up visits.

\section{Adverse events}

A total of eight nonserious AEs were reported during this study in three subjects including two study-related AEs, pain in extremity and seroma, and are shown in Table 2. All the study related AEs were resolved by end of the study. Investigators also reported three SAEs in three subjects including one that was study-related, a case of implant site dehiscence; and one subject had hepatic failure due to reasons not related to device or procedure after 6-month follow-up and died.

\section{Functioning, disability \& sleep}

PDI scores, a measure of pain interference with daily functioning, decreased from baseline after the initiation of stimulation (Figure 5A), and mean scores remained low through the final, 12-month follow-up visit. Similarly, subject function captured through GAF scores increased after the start of $10 \mathrm{kHz}$ SCS and remained elevated through the end of the study period (Figure 5B). Finally, pain interference with subject sleep also decreased, as quantified using PSQ-3 scores (Figure 5C). 
(A)

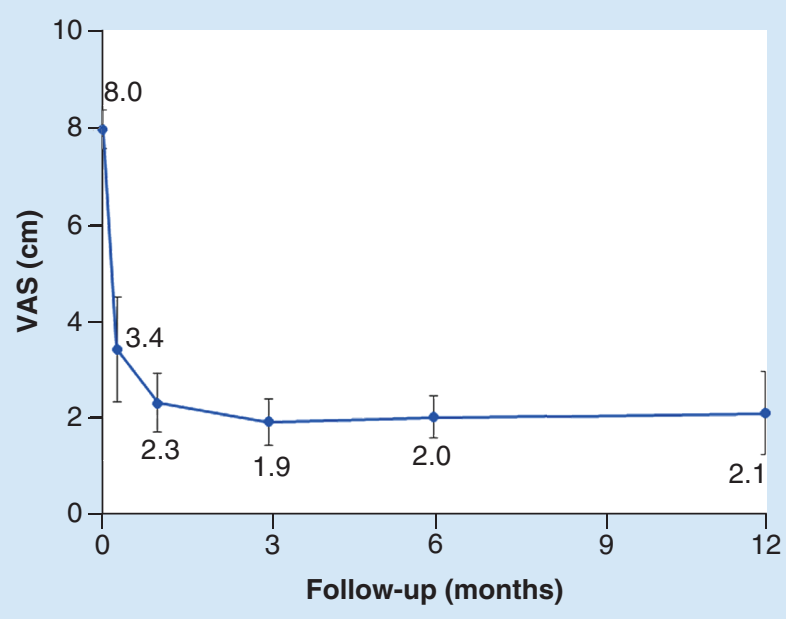

(B)

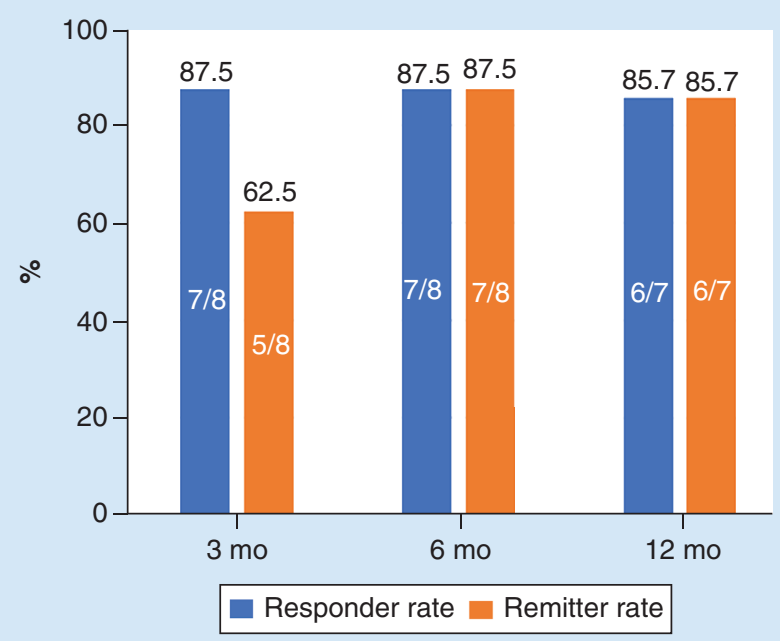

(c)

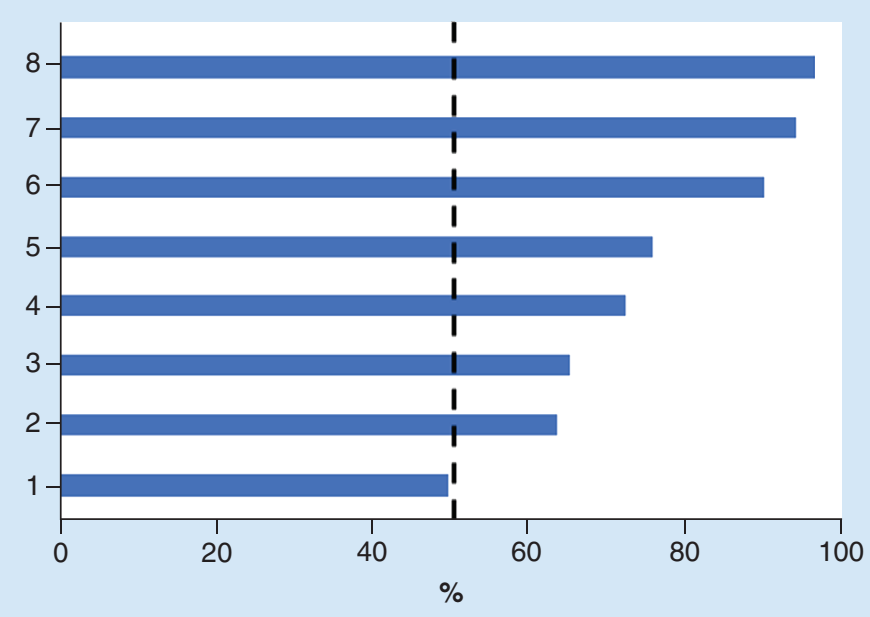

Figure 2. Subject-reported pain intensity decreased after the start of $10 \mathrm{kHz}$ SCS and remained low for the duration of the study. (A) Mean VAS scores are plotted against follow-up time. (B) Responder and remitter rates after 3, 6 and 12 months of stimulation. (C) Percentage pain relief at the 3-month follow-up shows seven of the eight subjects were responders $(\geq 50 \%)$.

SCS: Spinal cord stimulation; VAS: Visual analog scale.

\section{Perception of effectiveness}

Subjects' perception of the effectiveness of $10 \mathrm{kHz}$ SCS was assessed using subject-reported global impression of change, and after 3 and 12 months of stimulation, all subjects rated their pain better, with none reporting pain that was unchanged or similar to baseline (Figure 6A). The clinician assessments of these subjects included $88.9 \%$ judging subjects were at least 'moderately better' after 3 months of treatment, falling slightly to $81.3 \%$ at 12 months (Figure 6B).

\section{Discussion}

The data presented here support $10 \mathrm{kHz}$ SCS as a feasible treatment for subjects with PDN in the lower limbs. Decreases in subject-reported pain intensity were substantial, with mean pain relief of greater than $70 \%$. Pain reduction was reported within 1 week of stimulation and persisted through the 12-month period of stimulation. Studies of LF-SCS to treat painful PDN have reported pain relief of $50-60 \%[15,23]$. A review of seven randomized, controlled trials of pregabalin found more modest pain relief often limited by dose, up to $2.75 \mathrm{~cm}$ reductions in subjects taking $600 \mathrm{mg} /$ day [11]. In addition, these studies assessed subjects for 12 weeks after initiation, a much shorter period of follow-up than the 12 months included in the current study of $10 \mathrm{kHz}$ SCS. 


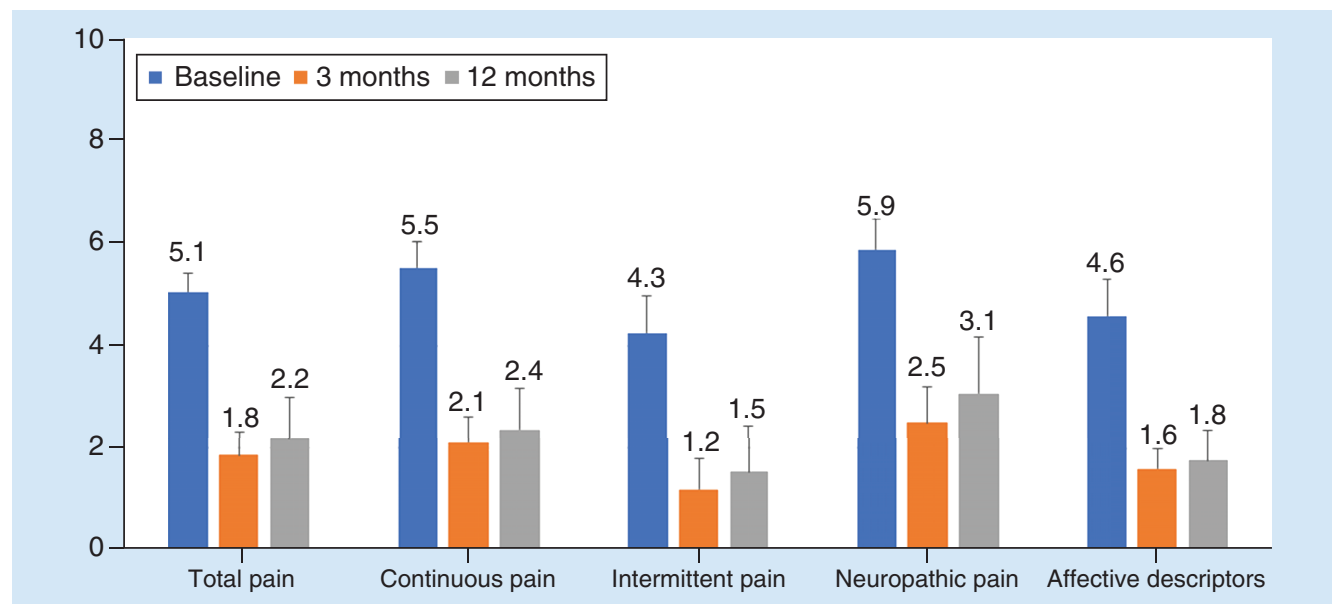

Figure 3. Pain scores reported using the SF-MPQ-2 decreased after 3 and 12 months of stimulation both overall and in each of the four pain subdomains.

(A)

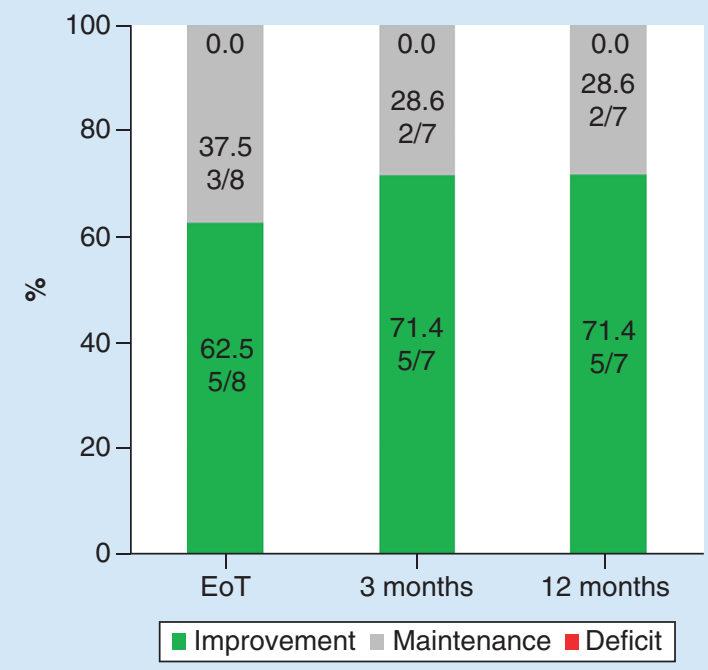

(B)

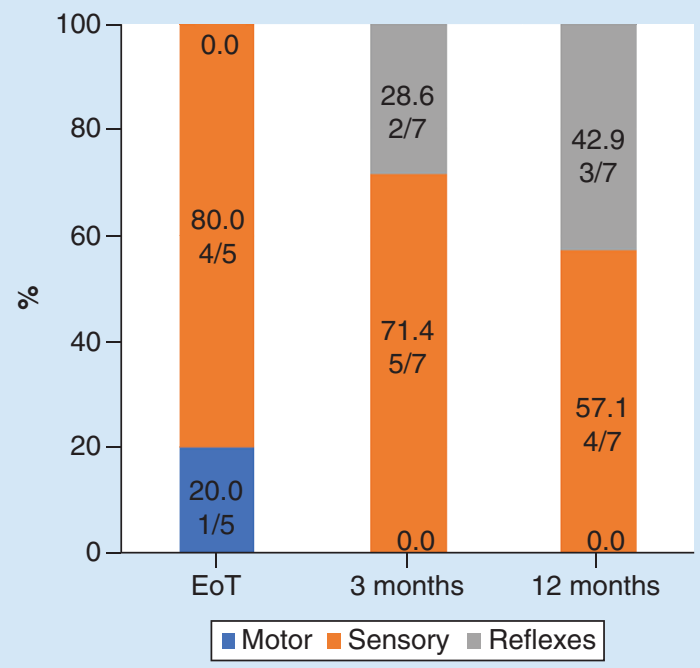

Figure 4. Neurological assessments showed improvements in some subjects' sensation and reflexes. (A) The neurological status of all subjects who received permanent implants was either improved or maintained after trial stimulation and after 3 or 12 months of stimulation. (B) The majority of improvements were in sensory and reflex functioning.

EOT: End of treatment.

The success rate of $10 \mathrm{kHz}$ SCS was high including eight of nine eligible subjects (89\%) who reported successful trial stimulations and six of seven subjects who were both responders (pain relief $\geq 50 \%$ ) and remitters (VAS $\leq 3.0 \mathrm{~cm}$ ) [24] at the 12-month follow-up. Previous studies in PDN subjects using LF-SCS reported a responder rates of $53 \%(\mathrm{n}=17)$ and $73 \%(\mathrm{n}=15)$ at 12 months [14,17]. The response rate seen in the current study was comparable with rates reported for $10 \mathrm{kHz}$ SCS in patients with back and leg pain, including responses in over $83 \%$ of subjects after 6 months and over $72 \%$ after 2 years in the SENZA-RCT [20,25]. Several smaller studies in back and leg pain reported response rates of up to $80 \%$ [26], and an analysis of real-world data from patients with back and leg pain found a 74\% response rate [27]. Likewise, patients with neck and arm pain had a response rate of $>80 \%$ response after 12 months of $10 \mathrm{kHz}$ SCS in a prospective clinical trial [28], and 78\% of subjects responded in a feasibility study in chronic abdominal pain [29].

Mean pain scores decreased in all four subdomains of the SF-MPQ-2 after the initiation of $10 \mathrm{kHz}$ SCS, including neuropathic pain and affective descriptors of pain. Pain interference with subject functioning, as assessed 

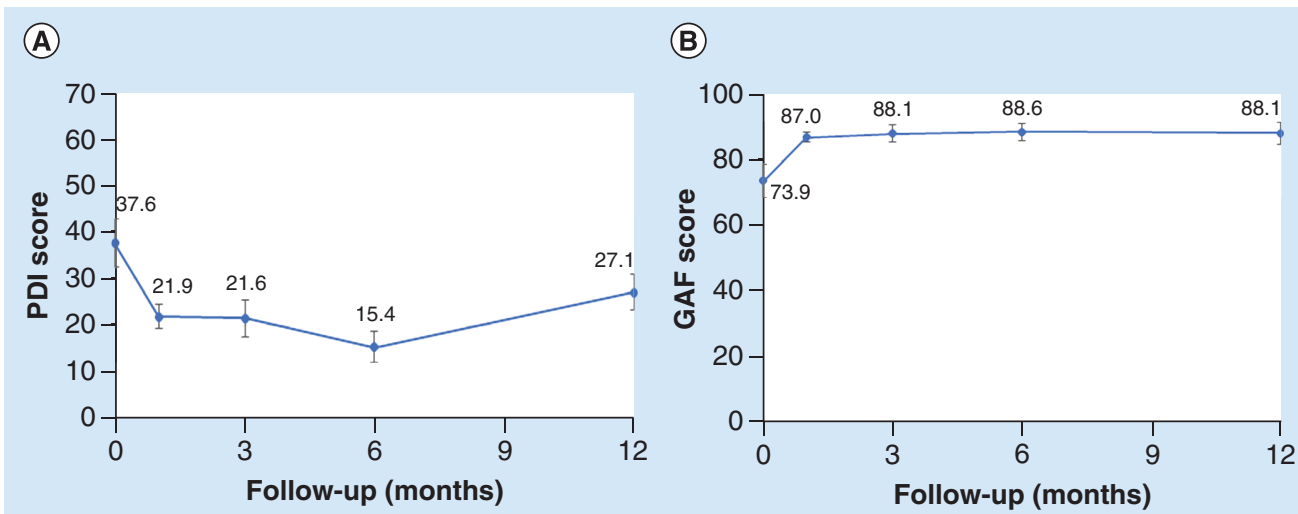

(C)

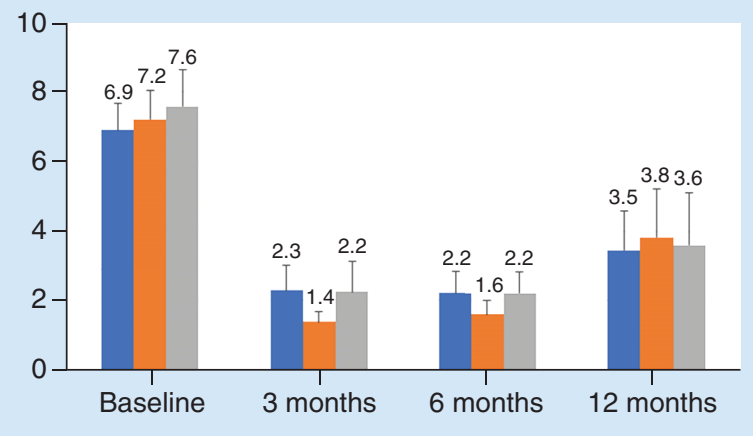

- How often did you have trouble falling asleep because of pain? - How often were you awakened by pain during the night? $\square$ How often were you awakened by pain in the morning?

Figure 5. Other measures of pain and pain interference with daily functioning also show durable improvements with $10 \mathrm{kHz}$ SCS. (A) Mean PDI scores decreased from baseline levels within 1 month of stimulation and remained low through the study period. (B) GAF scores increased within 1 month of stimulation and remained elevated through the 12-month follow-up. (C) Mean PSQ-3 sleep scores decreased after the initiation of stimulation.

GAF: Global assessment of functioning; PDI: Pain disability index; PSQ-3: Pain and sleep questionnaire; SCS: Spinal cord stimulation.
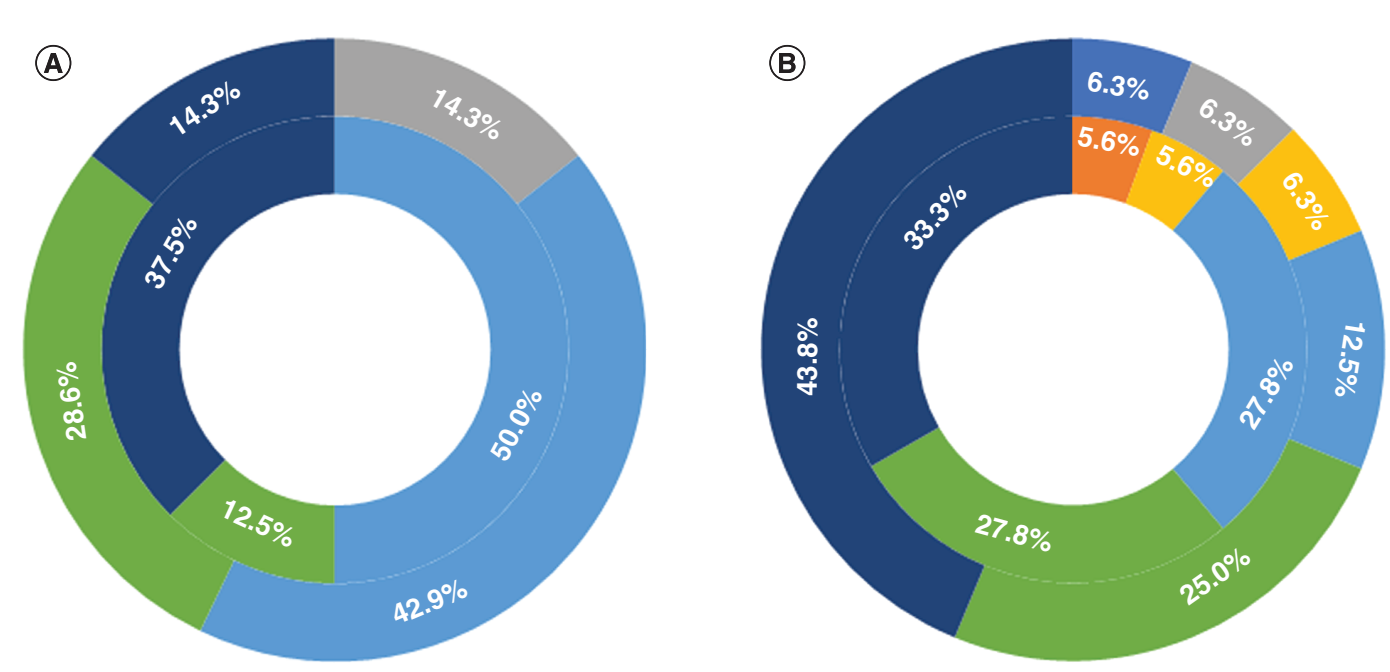

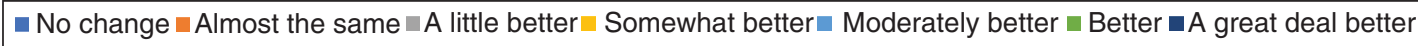

Figure 6. Improvement in Global Impression of Change following $10 \mathrm{kHz}$ treatment. Using the Global Impression of Change, both subjects (A) and clinicians (B) reported improvement in nearly all cases. 
by PDI and GAF, improved as well after the initiation of $10 \mathrm{kHz}$ SCS, showing that decreases in pain associated with stimulation translate to daily functioning as well. This was further supported by decreased pain interference with sleep demonstrated by the decline in mean PSQ-3 scores.

Pain is rarely the only symptom of PDN; subjects frequently report numbness and paresthesias [9]. In addition to pain relief, symptoms related to neurologic sensory and reflex deficits were improved after initiation of $10 \mathrm{kHz} \mathrm{SCS}$. Neurologic testing at baseline and during follow-up assessments showed that all subjects at least maintained baseline neurologic functioning and that most showed improvement at all time points after stimulation initiation. Most of the reported improvements were to sensory perception and reflexes, rather than motor improvements. Such sensory improvements have not been reported using other treatment methods and this result suggests that $10 \mathrm{kHz} \mathrm{SCS}$ may have unique beneficial effects for subjects with PDN. It is possible that the improved perfusion of the lower limbs with SCS could have contributed to sensory improvements [30]. Given that the previously investigated forms of SCS caused stimulation-induced paresthesia in the lower limbs that often overlap disease-induced paresthesia, it is likely that these neurological assessments were either not administered or improvements harder to notice in previous studies.

In addition to these promising results, $10 \mathrm{kHz}$ SCS is paresthesia-independent and offers several advantages over LF-SCS for treating PDN. Placement of the epidural leads for conventional SCS requires intra-operative paresthesia mapping to match the stimulation to the painful region. Anatomic placement of $10 \mathrm{kHz} S C S$ leads do not require paresthesia mapping allowing for procedural efficiency leading to patient safety and comfort [31]. Moreover, a predictable and shorter procedure time could potentially reduce the risk of infection at the surgical site, which poses an increased risk in patients with diabetes who undergo thoracolumbar surgery [32]. The paresthesia-free nature of $10 \mathrm{kHz}$ SCS also avoids the potential for painful or uncomfortable paresthesia that some subjects experience with LF-SCS [18]. Recent studies have shown the reduction of opioid intake with $10 \mathrm{kHz}$ SCS therapy may also help translate into supporting this modality in treating PDN where opioids are often used when other medications fail [33-36].

Safety data were also positive in this sub-analysis with two study-related AEs including one study-related SAE. None of the subjects required explant of the stimulation device due to infection, which was a significant concern in this population, and seven of eight subjects with a permanently implanted device continued through 12 months of the study. In contrast, about $77 \%$ of subjects with painful PDN treated with pregabalin will quit within 1 year due to side effects and low efficacy [37].

The current pilot study was limited by the post-hoc nature of the analysis and the small sample size. As a subanalysis, the source data were drawn from a larger study of subjects with polyneuropathies stemming from various etiologies, so assessments and measurements were not optimized for a study of subjects with PDN, and variables of interest, such as hemoglobin A1c levels of the subjects, were not assessed or recorded. The limited number of subjects in the analysis means it is difficult to assess whether the results are broadly applicable to patients with PDN. The study was also not powered to test the improvements in sensation. Findings from this study should therefore be carefully interpreted. Further controlled and real-world data are needed to further characterize the efficacy of $10 \mathrm{kHz}$ SCS in this population. To that end, a large randomized controlled trial has been initiated in this patient population to assess the long-term safety and efficacy of $10 \mathrm{kHz}$ SCS and is currently ongoing [38].

\section{Conclusion}

$10-\mathrm{kHz}$ SCS is a promising alternative therapy for patients with intractable painful PDN. There is a growing demand for alternative treatments for this common complication of diabetes, and high-frequency SCS offers the potential of substantial pain relief as well as improved sensory functioning.

\footnotetext{
Author contributions

V Galan, J Scowcroft, P Chang, S Li and P Staats were involved in data collection and interpretation of the results. J Subbaroyan was involved in design, conceptualization, analysis and preparing the outline for the manuscript. A Rotte contributed to the design, preparation of outline and worked with the medical writer in preparing the initial draft. All the authors have reviewed and approved the final version of this manuscript.
}

Financial \& competing interests disclosure

The study was funded by Nevro Corp. Vincent Galan is a consultant to Medtronic. James Scowcroft is a consultant to Nevro Corp. S $\mathrm{Li}$ is a consultant to Nevro Corp. P Staats is a consultant Medtronic, SPR therapeutics, electroCore, Nalu Sponsored research Nevro, 
Saluda. A Rotte and J Subbaroyan are employees of Nevro Corp. Medical writing support was provided by E MackLaren, Galen Medical Writing LLC (drafting the manuscript) and M Bhandaru (preparing the illustrations) and was funded by Nevro Corp. The authors have no other relevant affiliations or financial involvement with any organization or entity with a financial interest in or financial conflict with the subject matter or materials discussed in the manuscript apart from those disclosed.

No writing assistance was utilized in the production of this manuscript.

\section{Open access}

This work is licensed under the Attribution-NonCommercial-NoDerivatives 4.0 Unported License. To view a copy of this license, visit http://creativecommons.org/licenses/by-nc-nd/4.0/

\section{References}

Papers of special note have been highlighted as: $\bullet$ of interest; $\bullet \bullet$ of considerable interest

1. Centers for Disease Control and Prevention. National Diabetes Statistics Report, 2017: estimates of diabetes and its burden in the United States. (2017). www.cdc.gov/diabetes/data/statistics/statistics-report.html

2. Ogurtsova K, da Rocha Fernandes JD, Huang Y et al. IDF Diabetes Atlas: global estimates for the prevalence of diabetes for 2015 and 2040. Diabetes Res. Clin. Pract. 128, 40-50 (2017).

3. Schmader KE. Epidemiology and impact on quality of life of postherpetic neuralgia and painful diabetic neuropathy. Clin. J. Pain 18(6), 350-354 (2002).

4. Abbott CA, Malik RA, van Ross ER, Kulkarni J, Boulton AJ. Prevalence and characteristics of painful diabetic neuropathy in a large community-based diabetic population in the U.K. Diabetes Care 34(10), 2220-2224 (2011).

5. Sadosky A, Mardekian J, Parsons B, Hopps M, Bienen EJ, Markman J. Healthcare utilization and costs in diabetes relative to the clinical spectrum of painful diabetic peripheral neuropathy. J. Diabetes Complications 29(2), 212-217 (2015).

6. Callaghan BC, Cheng HT, Stables CL, Smith AL, Feldman EL. Diabetic neuropathy: clinical manifestations and current treatments. Lancet Neurol. 11(6), 521-534 (2012).

7. Mustapa A, Justine M, Mohd Mustafah N, Jamil N, Manaf H. Postural control and gait performance in the diabetic peripheral neuropathy: a systematic review. Biomed. Res. Int. 2016, 9305025 (2016).

8. Bril V, England J, Franklin GM et al. Evidence-based guideline: treatment of painful diabetic neuropathy: report of the American Academy of Neurology, the American Association of Neuromuscular and Electrodiagnostic Medicine, and the American Academy of Physical Medicine and Rehabilitation. Neurology 76(20), 1758-1765 (2011).

9. Snyder MJ, Gibbs LM, Lindsay TJ. Treating painful diabetic peripheral neuropathy: an update. Am. Fam. Physician 94(3), 227-234 (2016).

10. Finnerup NB, Attal N, Haroutounian S et al. Pharmacotherapy for neuropathic pain in adults: a systematic review and meta-analysis. Lancet Neurol. 14(2), 162-173 (2015).

- Systematic review documents number needed to treat data for pharmcotherapeutics.

11. Freeman R, Durso-Decruz E, Emir B. Efficacy, safety, and tolerability of pregabalin treatment for painful diabetic peripheral neuropathy: findings from seven randomized, controlled trials across a range of doses. Diabetes Care 31(7), 1448-1454 (2008).

12. Gibson W, Wand BM, O'Connell NE. Transcutaneous electrical nerve stimulation (TENS) for neuropathic pain in adults. Cochrane Database Syst. Rev. 14(9:9), CD011976 (2017).

13. de Vos CC, Rajan V, Steenbergen W, van der Aa HE, Buschman HP. Effect and safety of spinal cord stimulation for treatment of chronic pain caused by diabetic neuropathy. J. Diabetes Complications 23(1), $40-45$ (2009).

14. Slangen R, Pluijms WA, Faber CG, Dirksen CD, Kessels AG, van Kleef M. Sustained effect of spinal cord stimulation on pain and quality of life in painful diabetic peripheral neuropathy. Br. J. Anaesth. 111(6), 1030-1031 (2013).

15. de Vos CC, Meier K, Zaalberg PB et al. Spinal cord stimulation in patients with painful diabetic neuropathy: a multicentre randomized clinical trial. Pain 155(11), 2426-2431 (2014).

16. van Beek M, Geurts JW, Slangen R et al. Severity of neuropathy is associated with long-term spinal cord stimulation outcome in painful diabetic peripheral neuropathy: five-year follow-up of a prospective two-center clinical trial. Diabetes Care 41(1), 32-38 (2018).

17. van Beek M, Slangen R, Schaper NC et al. Sustained treatment effect of spinal cord stimulation in painful diabetic peripheral neuropathy: 24-month follow-up of a prospective two-center randomized controlled trial. Diabetes Care 38(9), e132-e134 (2015).

-• Randomized control trial (RCT) documents long-term efficacy of spinal cord stimulation (SCS).

18. De Carolis G, Paroli M, Tollapi L et al. Paresthesia-independence: an assessment of technical factors related to $10 \mathrm{kHz}$ paresthesia-free spinal cord stimulation. Pain Physician 20(4), 331-341 (2017).

19. Sills S. Treatment of painful polyneuropathies of diabetic and other origins with $10 \mathrm{kHz}$ SCS: a case series. Postgrad. Med. 9, 1-6 (2020). 
20. Kapural L, Yu C, Doust MW et al. Novel 10-kHz high-frequency therapy (HF10 Therapy) is superior to traditional low-frequency spinal cord stimulation for the treatment of chronic back and leg PainThe SENZA-RCT randomized controlled trial. Anesthesiology 123(4), 851-860 (2015).

-• Pivotal RCT demonstrates superiority of $10 \mathrm{kHz}$ SCS over traditional SCS.

21. North American Neuromodulation Society. Galan V, Chang P, Scowcroft J, Li S, Staats P, Subbaroyan J. (Eds). A prospective clinical trial to assess high frequency spinal cord stimulation (HF-SCS) at $10 \mathrm{kHz}$ in the treatment of chronic intractable pain from peripheral polyneuropathy. Presented at: The 22nd Annnual Meeting of the North American Neuromodulation Society; 2019. NV, USA, 17-19 January 2019.

22. Al-Kaisy A, Van Buyten J-P, Smet I, Palmisani S, Pang D, Smith T. Sustained effectiveness of $10 \mathrm{kHz}$ high-frequency spinal cord stimulation for patients with chronic, low back pain: 24-month results of a prospective multicenter study. Pain Medicine (Malden, Mass) 15(3), 347-354 (2014).

23. Pluijms WA, Slangen R, Bakkers $\mathrm{M}$ et al. Pain relief and quality-of-life improvement after spinal cord stimulation in painful diabetic polyneuropathy: a pilot study. Br. J. Anaesth. 109(4), 623-639 (2012).

24. Amirdelfan K, Gliner BE, Kapural L et al. A proposed definition of remission from chronic pain, based on retrospective evaluation of 24-month outcomes with spinal cord stimulation. Postgrad. Med. 131(4), 278-286 (2019).

25. Kapural L, Yu C, Doust MW et al. Comparison of 10-kHz high-frequency and traditional low-frequency spinal cord stimulation for the treatment of chronic back and leg pain: 24-month results from a multicenter, randomized, controlled pivotal trial. Neurosurgery 79(5), 667-677 (2016).

26. Sayed D, Kallewaard JW, Rotte A, Jameson J, Caraway D. Pain relief and improvement in quality of life with $10 \mathrm{kHz}$ SCS therapy: summary of clinical evidence. CNS (2020). https://onlinelibrary.wiley.com/doi/full/10.1111/cns.13285

-. Comprehensive review of $10 \mathrm{khHz}$ SCS efficacy.

27. Stauss T, El Majdoub F, Sayed D et al. A multicenter real-world review of $10 \mathrm{kHz}$ SCS outcomes for treatment of chronic trunk and/or limb pain. Ann. Clin. Transl. Neurol. 6(3), 496-507 (2019).

- $\quad$ Large retrospective study demonstrates real-world efficacy of $10 \mathrm{kHz}$ SCS.

28. Amirdelfan K, Vallejo R, Benyamin R et al. High-frequency spinal cord stimulation at $10 \mathrm{kHz}$ for the treatment of combined neck and arm pain: results from a prospective multicenter study. Neurosurgery https://doi.org/10.1093/neuros/nyz495 (2019) (Epub ahead of print).

29. Kapural L, Gupta M, Paicius R et al. Treatment of chronic abdominal pain with $10 \mathrm{kHz}$ spinal cord stimulation: safety and efficacy results from a 12-month prospective, multicenter, feasibility study. Clin. Transl. Gastroenterol. 11, e00133 (2020)

30. van Beek M, Hermes D, Honig WM et al. Long-term spinal cord stimulation alleviates mechanical hypersensitivity and increases peripheral cutaneous blood perfusion in experimental painful diabetic polyneuropathy. Neuromodulation 21(5), 472-479 (2018).

31. Kasapovic A, Rommelspacher Y, Gathen M et al. High-frequency spinal cord stimulation for the treatment of chronic low back and leg pain: implantation technique of percutaneous leads and implantable pulse generator. Arthrosc. Tech. 8(10), e1125-e1129 (2019).

32. Deng H, Chan AK, Ammanuel S et al. Risk factors for deep surgical site infection following thoracolumbar spinal surgery. J. Neurosurg. Spine 32(2), 292-301 (2019).

33. Al-Kaisy A, Van Buyten JP, Amirdelfan $\mathrm{K}$ et al. Opioid-sparing effects of $10 \mathrm{kHz}$ spinal cord stimulation: a review of clinical evidence. Ann. NY Acad. Sci. 1462(1), 53-64 (2019).

- Comprehensive review of studies shows opioid reduction in patients treated with $10 \mathrm{kHz}$ SCS.

34. Al-Kaisy A, Van Buyten JP, Carganillo R et al. $10 \mathrm{kHz}$ SCS therapy for chronic pain, effects on opioid usage: post hoc analysis of data from two prospective studies. Sci. Rep. 9(1), 11441 (2019)

35. DiBenedetto DJ, Wawrzyniak KM, Schatman ME, Kulich RJ, Finkelman M. $10 \mathrm{kHz}$ spinal cord stimulation: a retrospective analysis of real-world data from a community-based, interdisciplinary pain facility. J. Pain Res. 11, 2929-2941 (2018).

36. Salmon J. High-frequency spinal cord stimulation at $10 \mathrm{kHz}$ for widespread pain: a retrospective survey of outcomes from combined cervical and thoracic electrode placements. Postgrad. Med. 131(3), 230-238 (2019).

37. Yang M, Qian C, Liu Y. Suboptimal treatment of diabetic peripheral neuropathic pain in the United States. Pain Med. 16, 2075-2083 (2015).

38. Mekhail NA, Argoff CE, Taylor RS et al. High-frequency spinal cord stimulation at $10 \mathrm{kHz}$ for the treatment of painful diabetic neuropathy: design of a multicenter, randomized controlled trial (SENZA-PDN). Trials 21(1), 87 (2020). 